Archives de sciences sociales des religions

172 | octobre-décembre

Bulletin Bibliographique

\title{
Rebonds et questions
}

\section{François Trémolières}

\section{OpenEdition}

Journals

Édition électronique

URL : http://journals.openedition.org/assr/27224

DOI : $10.4000 /$ assr.27224

ISSN : 1777-5825

Éditeur

Éditions de l'EHESS

Édition imprimée

Date de publication : 1 octobre 2015

Pagination : 99-105

ISBN : 978-2-7132-2515-4

ISSN : 0335-5985

Référence électronique

François Trémolières, "Rebonds et questions », Archives de sciences sociales des religions [En ligne] 172 | octobre-décembre, mis en ligne le 26 avril 2018, consulté le 23 avril 2019. URL : http:// journals.openedition.org/assr/27224 ; DOI : 10.4000/assr.27224 


\section{François Trémolières}

\section{Rebonds et questions}

Rappelons l'occasion de ces quelques pages : une table ronde réunie par Pierre Antoine Fabre, le 12 novembre 2013, pour saluer la parution du tome II de La Fable mystique. Dominique Julia et Jacques Le Brun, historiens du catholicisme moderne - et pour J. Le Brun plus particulièrement de la spiritualité -, ont tous deux bien connu Michel de Certeau, cheminé avec lui dans leur travail ; D. Julia a écrit avec M. de Certeau et Jacques Revel un article resté fameux sur la notion de culture populaire : "La beauté du mort " (Politique aujourd'hui, 1970), et un ouvrage sur le rapport Grégoire (Une politique de la langue, Gallimard, 1975). Patrick Goujon et Jocelyne Sfez sont d'une génération postérieure. Jésuite, spécialiste de Surin, P. Goujon n'a cessé de se confronter à l'œuvre certalienne, en particulier les travaux d'édition et d'histoire des débuts de la Compagnie de Jésus en France. J. Sfez, éditrice des Conjectures de Nicolas de Cues (Beauchesne, 2011), l'abordait au contraire pour la première fois - le chapitre sur le Cusain constituant l'apport le plus notable de ce tome II. J'étais chargé d'introduire à la discussion avec le public (nombreux) en livrant quelques réflexions improvisées, suscitées par ces quatre communications. Les ASSR ayant eu l'idée de recueillir celles-ci pour une publication, j'essaierai d'inviter le lecteur à un prolongement qui lui soit personnel, en indiquant quelques thèmes à partir de ma lecture de leurs versions écrites, avec le même caractère de spontanéité.

\section{L'œuvre et son rapport au livre}

Le premier est le motif de l'œuvre, en effet, placé par Dominique Julia en ouverture de son propos. Désignons d'abord par là l'ensemble des écrits certaliens, dont D. Julia rappelle la variété des champs qu'ils ont couverts, partant l'extension des réceptions possibles, ou du moins des milieux de réception, encore augmentée par les aléas d'une diffusion en partie posthume, et qui n'a pas pris les mêmes chemins selon les pays, c'est-à-dire selon les langues d'accueil (les traductions).

Mais le terme peut s'entendre aussi autrement, comme ce qui est désiré dans l'écriture - et d'un désir travaillé par la mort. Citant Blanchot, Certeau commente 
l'écart entre l'œuvre et le livre ${ }^{1}$ : le livre est là, mais l'œuvre est absente, " dissimulée en tout cas, offusquée par l'évidence du livre, derrière laquelle elle attend la décision libératrice, le Lazare, veni foras " (L'espace littéraire). La "lecture absolue " dont il a voulu faire la théorie - absolue en ce sens qu'elle est "déliée du texte et que, de ce fait, elle s'absout de sa loi ${ }^{2}$ » - conçoit le livre comme une sorte de tombeau dont une sorte de corps pourrait sortir: une voix, "la voix ou l'œuvre enterrée dans le livre ». Ce " désir de la Voix » dépasse le « livreobjet » en des pratiques de lecture que Certeau identifie à celles des "spirituels » mais aussi, très explicitement, à " une pratique moderne du livre ", contemporaine, dont Blanchot offre l'une des « figures ». Inlassable lecteur de lui-même, l'auteur Certeau travaille à son propre effacement, c'est-à-dire à la capture de sa voix dans le livre.

Luce Giard rappelle la reprise incessante du texte, Certeau remaniant, transformant, continuant de réécrire jusque sur épreuves, de sorte qu'il en « retardait [différait] l'achèvement ${ }^{3}$ ». Le livre est ainsi, nécessairement, un compromis. Et l'acceptation de ce compromis, dès lors que l'auteur n'est plus là pour l'assumer et en décider, relève, comme l'écrit encore D. Julia, d'un "travail de deuil ». Il faut accepter que la tâche " est impossible à toute autre personne ${ }^{4}$ ", et que cette personne est morte ; accepter, pour faire un livre, l'impossibilité de faire œuvre, et que le «corps» du livre soit de fragments. Ce travail a sa temporalité, cette acceptation aura pris une trentaine d'années.

Parce que D. Julia nous invite, à la toute fin de son étude, à un parallèle avec l'abbé Bremond, qu'il soit permis de souligner ici que le même écart sépare les deux tomes de La Fable mystique - et donc nous sépare du premier (1982) que celui qui tenait Certeau à distance de l'Histoire littéraire du sentiment religieux en France (dernier volume paru en 1933) lorsqu'il fit de son auteur - un peu plus éloigné il est vrai de sa disparition -, à l'occasion d'un colloque de Cerisy (1965), l' " historien d'une absence ». D. Julia lit « un refus net de la voie choisie par Bremond " dans le fameux incipit du tome I de La Fable mystique: «Ce livre se présente au nom d'une incompétence. Il est exilé de ce qu'il traite. L'écriture que je dédie aux discours mystique de (ou sur) la présence (de Dieu) a pour statut de ne pas en être. " Un lecteur de Bremond pourrait être sensible au contraire à ce qui fait écho à celui de l'Histoire littéraire... ${ }^{5}$ (le dernier paragraphe des "notes liminaires ») : " quant au témoin de ces témoins, quant au scribe lui-même, il est semblable à un calligraphe copiant avec amour des chefsd'œuvre qu'il n'entend point... ». Au-delà des précautions qui encombrent sans

1. La Fable mystique II, p. 217.

2. Ibid., p. 197.

3. «Présentation", op. cit., p. 11.

4. Ibid., p. 10.

5. Voir en ce sens, tout récemment, Jean-Pierre Jossua, "En relisant Bremond ", Revue des sciences philosophiques et théologiques, t. 98 n 4, octobre-décembre 2014, spéc. p. 736. 
doute ce début de l'opus magnum bremondien (mais sont-elles à dédaigner pour qui a lu l'introduction générale aux Arts de faire, et son éloge de la «tactique » ?), il est permis d'y entendre aussi un aveu, que Certeau à sa façon redoublerait, réfléchirait : il s’agit bien du rapport à une expérience, mais manquante.

\section{Mystique et histoire}

Dans les termes du P. Bourgoing par exemple (sa préface aux CEuvres de Bérulle, 1644), la " théologie mystique " s'autorise de l'expérience, là où la dogmatique s'autorise de la raison et la positive de la Révélation. Or, en indiquant que la notion de "science expérimentale" - centrale chez Surin, et dans la conception que nous nous faisons à sa suite, en lecteurs de Certeau, de la mystique "moderne »- est "si importante » chez Nicolas de Cues (mais, préciset-elle plus loin, en un sens autre que Surin), Jocelyne Sfez met d'emblée l'accent sur ce qui paraît un impensé de La Fable mystique : le rapport de "la mystique » (cette mystique moderne, identifiée par l'apparition du substantif, autour de 1600) à ce qui précède. Elle au contraire crédite Certeau d'avoir attribué au Cusain un "rôle décisif dans la mystique chrétienne "; elle pointe dès La Fable mystique I des indices d'inscription de la mystique dans une plus longue durée - nuançant cependant ce sur quoi Certeau insistait, la "référence dominante» à Denys depuis le XIII ${ }^{\mathrm{e}}$ siècle, dont le Cusain aurait été un relais.

L'inédit sur le De Icona (1453) donné en chapitre 1 de La Fable mystique II, plus riche d'informations historiques que le texte plus court que Certeau lui avait consacré de son vivant (dans Traverses, en 1984), ouvre donc la voie à une lecture plus médiéviste, moins moderniste, de son œuvre - Alain Boureau avait déjà souligné la concomitance entre L'Écriture de l'histoire (1975), c'est-à-dire l'apport épistémologique de Certeau à la discipline historique, et le renouveau de l'histoire des doctrines et de la philosophie médiévales ${ }^{6}$. Ce qui est en jeu, selon J. Sfez, c'est " la thèse d'un Nicolas de Cues mystique » (et donc aussi ce que l'on entend par « mystique »). Elle reprend les critiques de Kurt Flasch envers un qualificatif qui lui paraît anachronique, une qualification construite sur une dichotomie artificielle entre "scolastique » et "mystique », ignorante en particulier de l'œuvre latine (savante) de Maître Eckhart et abusivement confortée par la condamnation de ce dernier et sa postérité rhénane. La posture " moderne » de Certeau le protégerait de cet anachronisme.

Le résultat, paradoxal, est que Certeau permettrait de comprendre à la fois en quoi Cues ne peut être dit mystique, et «la fécondité de sa pensée pour le discours mystique ». J. Sfez accorde ainsi Certeau et Flasch pour les opposer à Alain de Libera et Frédéric Nef qui, dans une longue étude de La Fable mystique

6. Dans sa contribution à Lire Michel de Certeau, Michel de Certeau lesen, dir. Philippe Büttgen et Christian Jouhaud, Zeitsprünge, XII, 1/2, 2008, p. 115. 
publiée en 1983, assez polémique, avaient entendu montrer que Certeau s'interdisait en réalité de penser une histoire de la mystique, en n'ayant retenu de l'insistance de la tradition dionysienne au moment critique de la Renaissance que l'indice d'un tournant langagier, et oublié ou rejeté la persistance de la « question de l'Un " durant les siècles qui avaient précédé, notamment en contexte germanique. Sans entrer ici dans une querelle dont je n'ai pas la compétence, je me demande néanmoins si la prise en compte de cette dimension hénologique ${ }^{7}$ ne serait pas la condition pour aborder l'œuvre d'un Eckhart d'abord comme philosophique (ou d'abord en historien de la philosophie) et donc si Flasch ne serait pas plus proche ici de Libera (et Nef) que de Certeau (qui, rappellent Libéra / Nef, analyse le volo eckartien en terme de "contrat énonciatif ${ }^{8}$ " et non comme acte spéculatif) ?

On ajoutera que Certeau lui-même a revendiqué 9 une "hétérologie " du "discours historique », par opposition au "discours philosophique » défini comme " hénologique » en quelque sorte par essence.

Quoi qu'il en soit, il concédait à ses adversaires que "les rapports entre la mystique dite "métaphysique" (de tradition eckartienne) et celle qu'on a appelée tour à tour "nuptiale" ou "psychologique" ", ou encore affective, restent à penser (ou « repenser») - entre " une mystique ontologique médiévale et une mystique liée à la rhétorique de la Renaissance ». Il me semble qu'il s'agit là d'une question qu'il nous a léguée, davantage que d'une piste qu'il a explorée : ce qu'il fit et qu'il a "voulu ", selon ses propres termes, c'est " analyser, sous sa figure mystique, ce transit vers une conception moderne $\left(\mathrm{XVI}^{\mathrm{e}}-\mathrm{XVII}{ }^{\mathrm{e}}\right.$ siècle) du sujet, du langage et de la science " ${ }^{10}$. L'importance historique du De Icona aurait été d'avoir précédé, si l'on comprend bien, d'avoir annoncé le transfert " moderne » du doctrinal au formel (comme aux pratiques); de pouvoir l'opérer pour son lecteur et d'avoir su lui assigner son lieu : «la mystique ».

\section{Certeau jésuite : théologie, herméneutique, inscription ecclésiale}

Patrick Goujon reprend à son compte l'analyse certalienne de la mystique comme énonciation, mais pour la conduire à une conclusion qui prend nettement le contrepied de celles de Certeau, en affirmant que le "sens de l'autre " dans le conversar mystique "n'est pas celui d'une absence mais celui d'une présence ».

7. De « hénologie », étymologiquement « science de l'Un ». Sur l'emploi (critique) du terme par Certeau, voir infra et références note 9.

8. Cf. La Fable mystique I, p. 225.

9. Dans la conclusion à L'absent de l'histoire (1973), p. 173-177. "Contre une réduction hénologique ", voir aussi «La rupture instauratrice " (1971), in La faiblesse de croire, p. 216.

10. Littoral $\mathrm{n}^{\circ} 9$, juin 1983, p. 116, en réponse à A. de Libera et F. Nef, "Le discours mystique. Problèmes d'histoire et de méthode ", dans le dossier "La mystique : fable ou discours? » que cette « revue de psychanalyse » (son surtitre) avait consacré à La Fable mystique. 
Une telle divergence engage une discussion de fond qui excède de beaucoup les limites de mon propos. P. Goujon ne l'exprime pas en termes théologiques, au sens disciplinaire du terme, mais elle aboutit bien à un discours sur Dieu (théologie) : affirmation de Sa présence, qui révèle en creux l'enjeu de foi dans l'entreprise certalienne.

Il serait possible de montrer en quoi le motif de l'absence serait compatible chez Certeau - et même sa plus juste expression - avec la croyance. La revendication de scientificité dans ses écrits, et d'une scientificité qui s'identifie à la formalité (comme déjà chez Cues ou du moins dans la lecture virtuose qu'il en donne), va de pair avec un discrédit des "contenus » et plus largement de la prétention de vérité, ce qu'il appelle "la tromperie du sens " ${ }^{11}$. C'est que le je de l'expérience est toujours pris dans le nous d'un langage, selon une formule de Certeau dans l'article sur Surin "interprète " de Jean de la Croix ${ }^{12}$, cité à la fois par P. Goujon et Jacques Le Brun. "L'auteur ou le sujet, poursuit-il, n'apparaît que soumis déjà à la loi d'une communauté. [...] Tout écrit est donc de structure “ecclésiale". » Langage implique communauté - de même pourrait-on dire, vérité implique Église : il y a un devenir institution de la vérité. L'historien marque, par l'étude de successifs "états du texte ", l'écart objectif d'avec une origine inatteignable, irrémédiablement passée, qui oriente pourtant tout son travail ; au lieu que le croyant fait le chemin inverse : refaire "événement ", surmonter l'aliénation au collectif (ecclesia) par une "prise de parole " qui l'en libère en tant que sujet, sur un mode à la fois d'appartenance et de différence ${ }^{13}$.

Il faudrait suivre les rares indices d'un débat de Certeau avec les théologiens de son temps, aussi avec l'herméneutique - plutôt rudement traitée dans le passage du chapitre sur la glossolalie cité ici par D. Julia ${ }^{14}$ (interpréter, c'est prendre le parti du sens contre celui de la parole). Pourtant le projet herméneutique est de ceux qui prennent au sérieux l'historicité de la foi chrétienne, ce qui fit écrire à Schleiermacher (dont le nom n'apparaît qu'une fois dans l'index des deux volumes de La Fable mystique) que « le but de la théologie consiste à exposer toujours plus purement l'essence particulière du christianisme dans chaque instant à venir ${ }^{15}$ »; et l'on peut se demander s'il n'y aurait pas là, malgré tout, matière à un dialogue.

11. La Fable mystique II, p. 344 (voir la longue citation de ce passage que donne D. Julia, sur la glossolalie).

12. Repris comme chapitre 4 de La Fable mystique II, ici p. 167.

13. Le piège serait de penser un "dehors" : voir par exemple la conclusion déjà citée (l'opposition hénologie / hétérologie) de L'absent de l'histoire, les considérations du Christianisme éclaté (1974) sur le "corps ecclésial " (cf. "Du corps à l'écriture, un transit chrétien ", in La faiblesse de croire, 1987) et l'opposition tactique / stratégie (le rapport au "propre") dans Arts de faire, elle aussi mentionnée plus haut.

14. Op. cit., p. 344-345, voir supra note 11. Moins rudement dans le chapitre 4, voir l'allusion à Gadamer, p. 164.

15. Le statut de la théologie (1811), $\mathbb{S} 84$; d'après Christoph Theobald, Le christianisme comme style, Paris, Cerf, 2007, t. I, p. 26. 
La Fable mystique II aborde plus frontalement la question de l'exégèse, surtout dans le chapitre consacré à "l'érudition biblique " ${ }^{16}$. Mais c'est aux origines mêmes du projet certalien qu'on la trouve, si l'on se souvient de la recension qu'a faite Certeau, dans la Revue d'ascétique et de mystique, en $1960^{17}$, des deux premiers tomes de la somme de Lubac, Exégèse médiévale. Les quatre sens de l'Écriture: l'exégèse y est conçue non comme "science auxiliaire à la théologie " mais comme "la théologie même - et plus que la théologie, si l'on n'étend pas la signification du mot jusqu'à la spiritualité »; "point essentiel » de la méthode de Lubac, l'attention au langage, ce que Certeau reprend et accentue (au point de noter l'amorce d'une divergence : " le problème du langage est présent à toute l'œuvre du P. de Lubac, mais il reste, semble-t-il, sans solution nette $\left.^{18} »\right)$. Il signale l'ouvrage et sa recension au début de la deuxième partie de La Fable mystique I : "Une topique ", avant d'entamer le récit (et l'analyse) de l'apparition du substantif "mystique ", faisant d'emblée référence à un autre grand livre de Lubac: "De sa carrière médiévale [du mot "mystique"], il suffira de rappeler une étape, relative à l'expression "corpus mysticum” (corps mystique). Ce n'est qu'un “cas” entre d'autres, mais il présente l'avantage d'avoir été l'objet d'une minutieuse analyse théologique dont mon histoire pourrait être la suite ${ }^{19}$."

Je vois là, et donc dans le rapport de Certeau à Lubac (dont la rencontre, lors des années de séminaire à Lyon, pourrait avoir décidé de son entrée dans la Compagnie de Jésus), une clé de lecture de La Fable mystique. Je l'indique comme une piste sur laquelle il faudra revenir, ajoutant seulement que ce qui se donne ici comme "suite» marque aussi bien une torsion, puisque l'on passe du "théologique » à "l'histoire ». Lorsque Certeau, dans "La rupture instauratrice " (son texte le plus tranchant quant à sa relation à la théologie comme discipline), se revendiquera explicitement du modèle exégétique mis en place par Lubac ( «la conversion de l'Ancien Testament au Nouveau Testament ${ }^{20}$ »), celui-ci refusera vigoureusement de s'y reconnaître, parce qu'il en fait un " progrès d'absence ", aboutissant à la perte de tout "contenu religieux " et pour finir l'impossibilité d'un objet Église ${ }^{21}$.

16. Op. cit., p. 287 et suiv., sur Richard Simon et Le Maistre de Sacy.

17. No 143 (vol. 36), juillet-septembre 1960, p. 358-371.

18. Article cité, note 29 , p. 369.

19. La Fable mystique I, p. 107-108 (référence à Corpus mysticum, $2^{e}$ éd. 1949).

20. Repris, in La faiblesse de croire, p. 222.

21. Voir La postérité spirituelle de Joachim de Flore, Paris, Lethillieux, 1981, t. II, p. 447449 ; et déjà Les Églises particulières dans l'Église universelle, Paris, Aubier, 1971, note p. 55, soit en réaction quasi immédiate à la publication de «La rupture instauratrice » dans la revue Esprit (juin 1971). 


\section{Questions de style}

J. Le Brun nous rend sensibles à la dimension « littéraire » de l'histoire telle que la pratique Certeau, non au sens de Bremond (sinon qu'il s'agit d'une histoire adossée à des écrits), mais par l'attention à la « littérarité ». On l'a vu, le " dire » l'emporte sur le dit, l'énonciation sur l'énoncé (d'où le recours à la sémiotique), l'acte sur son résultat, la parole sur le savoir. Cela suppose une attention particulière à la dimension non sémantique de la langue, musicale, poétique - et, comme l'indique aussi D. Julia, c'est ainsi qu'il faut entendre l'emploi du mot "fable» (selon l'étymologie fari) par Certeau.

Il y a dans ce rapport du dire au dit un travail de la négation, qui fait suggérer à J. Le Brun, à la suite de L. Giard, une influence de Hegel sur Certeau. Je prolongerai sa remarque en signalant la lecture à mes yeux très convaincante de l'un des textes majeurs de L'écriture de l'histoire: "La formalité des pratiques ", par Philippe Büttgen, dans le volume franco-allemand Lire Michel de Certeau qu'il a dirigé avec Christian Jouhaud en $2008^{22}$. Ce que décrit Certeau, à savoir le passage, selon le sous-titre de son étude, "du système religieux [XVII ${ }^{e}$ siècle] à l'éthique des Lumières $\left[\mathrm{XVIII}^{\mathrm{e}}\right]$ ", c'est l'histoire, commente Ph. Büttgen, d'une " opération de la société sur elle-même ", dont le moteur est la transformation du rapport à la vérité, en sorte que la sécularisation devient (à partir d'une "instance du doctrinal pur» qui serait le Moyen Âge) «la résultante d'une compréhension transformée de la vérité »: entreprise historiographique dans laquelle $\mathrm{Ph}$. Büttgen propose de lire «l'une des plus puissantes réactivations de l'idéalisme survenue dans le champ de la pensée contemporaine »...

Ce caractère littéraire passe enfin, peut-être d'abord, par une attention à la manière des auteurs étudiés - les «tours » selon Pascal - et la production d'une écriture propre : " un style particulier d'historien ", avait écrit L. Giard en ouverture du Lieu de l'autre (2005). Certeau à la dernière page de l'introduction à La Fable mystique II, appliquant la notion aux mystiques qu'il étudie, se réfère comme elle à Gilles-Gaston Granger (Essai d'une philosophie du style, 1968) - lequel traitait de mathématiques. "Styles scientifiques ", "styles mystiques ", Certeau note qu'ils sont « indissociables d'une esthétique ${ }^{23}$ ». J. Le Brun relève dans le chapitre inaugural sur Nicolas de Cues son insistance là encore sur un "style " - des " gestes ", une « façon de faire » - et montre ce qui le rapproche du style certalien, au point que ce texte lui paraît quasi "autobiographique ", non par la forme de l'aveu bien sûr (qui précisément lui serait infidèle) mais par son tour réflexif et spéculatif, si singulier. Et c'est cette singularité, qui s'affirme dans l'art du commentaire, du dédoublement et de l'appropriation, qu'il nous reste à méditer.

François TRÉMOLIÈRES

Université Paris Ouest Nanterre La Défense francois.tremolieres@u-paris10.fr

22. "Le contraire des pratiques. Commentaires sur la doctrine de Michel de Certeau ", op. cit., p. 69-97.

23. Op. cit., p. 50. 
